\title{
Numerical Methods for Pricing American Options with Time-Fractional PDE Models
}

\author{
Zhiqiang Zhou' ${ }^{1}$ and Xuemei Gao ${ }^{2}$ \\ ${ }^{1}$ School of Economic Mathematics, Southwestern University of Finance and Economics, Chengdu, Wenjiang 611130, China \\ ${ }^{2}$ School of Economic Mathematics and School of Finance, Southwestern University of Finance and Economics, Chengdu, \\ Wenjiang 611130, China
}

Correspondence should be addressed to Zhiqiang Zhou; zqzhou@2014.swufe.edu.cn

Received 26 September 2015; Accepted 22 December 2015

Academic Editor: George Tsiatas

Copyright (C) 2016 Z. Zhou and X. Gao. This is an open access article distributed under the Creative Commons Attribution License, which permits unrestricted use, distribution, and reproduction in any medium, provided the original work is properly cited.

In this paper we develop a Laplace transform method and a finite difference method for solving American option pricing problem when the change of the option price with time is considered as a fractal transmission system. In this scenario, the option price is governed by a time-fractional partial differential equation (PDE) with free boundary. The Laplace transform method is applied to the time-fractional PDE. It then leads to a nonlinear equation for the free boundary (i.e., optimal early exercise boundary) function in Laplace space. After numerically finding the solution of the nonlinear equation, the Laplace inversion is used to transform the approximate early exercise boundary into the time space. Finally the approximate price of the American option is obtained. A boundary-searching finite difference method is also proposed to solve the free-boundary time-fractional PDEs for pricing the American options. Numerical examples are carried out to compare the Laplace approach with the finite difference method and it is confirmed that the former approach is much faster than the latter one.

\section{Introduction}

Fractional differential equations have wide applications in the fields of physics modelling (see, e.g., a book [1] and the references therein). Based on the fact that the fractional-order derivatives are characterized by the "globalness" and can provide a powerful instrument for the description of memory and hereditary properties of different substances, recently the fractional differential equations are applied to the area of mathematical finance by generalizing the Black-Scholes (BS) equations to the fractional order. In this subject, there are mainly two types of fractional derivatives involved: a spacefractional derivative and a time-fractional derivative. Regarding a space-fractional derivative, Carr and $\mathrm{Wu}$ [2] introduced a finite moment log stable (FMLS) model and showed that the model outperforms other widely used financial models. Cartea and del-Castillo-Negrete [3] successfully connected the FMLS process with the space-fractional derivatives. Later on, Chen et al. [4] derived the explicit closed-form formula for European vanilla options under the FMLS model. As for time-fractional derivative, Wyss [5] derived a closed-form solution for European vanilla options under a time-fractional B-S equation. Kumar et al. [6] further explored the European option pricing under time-fractional B-S equation using the Laplace transform. Later on using tick-to-tick date, Cartea [7] found that the value of European-style options satisfies a fractional partial differential equation with the Caputo timefractional derivative. Jumarie [8] derived a time-and-spacefractional B-S equation. Liang et al. [9] introduced bifractional B-S models in which they assume that the underlying asset follows a fractional Itô process and the change of option price with time is a fractal transmission system. Chen et al. [10] derived an analytic formula for pricing double barrier options based on a time-fractional B-S equation.

To the best of our knowledge, we are only aware of one paper Chen et al. [11] studying the American option pricing under fractional derivatives. In [11], they studied predictorcorrector finite difference methods for pricing American options under the FMLS model which is a kind of spacefractional derivative model. In this paper we study the pricing of American options with time-fractional model which has essential difference to the space-fractional model. Following 
the model in [10], we assume that the underlying asset price still follows the classical Brownian motion, but the change in the option price is considered as a fractal transmission system. The price of such American option follows a time-fractional partial differential equation (PDE) with free boundary. The solution of the time-fractional PDE with free boundary is much more challenging than solving the fractional PDEs with fixed boundary for European option pricing in [10]. In this paper we develop two numerical methods, Laplace transform method and finite difference method, to solve the free-boundary problem of time-fractional PDE. The Laplace transform for partial differential equation can result in an ordinary differential equation; that is, it can convert partial differential equations into ordinary differential equations. However the solution of ordinary differential equations is much simpler than that for partial differential equations. This is the intuition of using Laplace transform method for solving the PDEs with free boundary. The Laplace transform method has been successfully applied to the pricing American options with classical (integer) B-S equations (see [12]). In the current work, by applying the Laplace transform to time-fractional PDE with respect to time, constructing the general solutions to the resulted ordinary differential equation (ODE), and then using the (free) boundary conditions, we derive a nonlinear equation for the free boundary (or optimal early exercise boundary) function in Laplace space. This nonlinear equation is solved by the secant method and the approximate free boundary in the Laplace space is obtained. Then the Laplace inversion is applied to get the optimal early exercise boundary in the time space. Finally the approximate value of the American option is obtained. Also motivated by $[13,14]$ for solving integer-order PDEs with free boundary, we develop a boundary-searching finite difference method to solve the time-fractional PDEs free boundary. The comparison of the Laplace method with the finite difference method is made by several examples. The numerical results show that the Laplace transform method is much more efficient than the finite difference method.

The rest of paper is arranged as follows: In Section 2 we describe the American option pricing models with timefractional derivatives; in Section 3 we introduce the Laplace transform methods for the problem; in Section 4 we give the boundary-searching finite difference methods for the problem; in Section 5 we provide numerical examples to compare the performance of the Laplace transform method with FDM; the conclusions are made in the final section.

\section{American Option Pricing Models with Time-Fractional Derivatives}

Assume that the underlying asset price is governed by the constant elasticity of variance (CEV) model (see, e.g., Cox [15]):

$$
d S_{t}=(r-q) S_{t} d t+\delta S_{t}^{\beta+1} d W_{t}
$$

where $r$ is the risk-free interest rate, $q$ is the dividend yield, and $W_{t}$ is standard Brownian motion. $\sigma(S)=\delta S^{\beta}$ represents the local volatility function and $\beta$ can be interpreted as the elasticity of $\sigma(S)$. If $\beta=0$, then SDE (1) becomes the lognormal diffusion model.

Denote by $f(S, t)$ the price of American option with $S$ being underlying and $t$ being the current time. From SDE (1), $f(S, t)$ should satisfy the modified B-S equation

$$
\frac{\partial f}{\partial t}+\frac{1}{2} \delta^{2} S^{2 \beta+2} \frac{\partial^{2} f}{\partial S^{2}}+(r-q) S \frac{\partial f}{\partial S}-r f=0 .
$$

However, it is argued that the time derivative $\partial f / \partial t$ should be replaced by the fractional derivative $\partial^{\alpha} f / \partial t^{\alpha}(0<\alpha<$ 1) under the assumption that the change in the option price follows a fractal transmission system (see, e.g., $[9,10])$; that is,

$$
\frac{\partial^{\alpha} f}{\partial t^{\alpha}}+\frac{1}{2} \delta^{2} S^{2 \beta+2} \frac{\partial^{2} f}{\partial S^{2}}+(r-q) S \frac{\partial f}{\partial S}-r f=0
$$

The meaning of the assumption is that the diffusion of the option price depends on the history of the time to maturity. Moreover it is suggested by $[9,10]$ that the following righthand side modified Riemann-Liouville derivative can be used:

$$
\begin{aligned}
\frac{\partial^{\alpha} f}{\partial t^{\alpha}}=\frac{1}{\Gamma(1-\alpha)} \frac{\partial}{\partial t} \int_{t}^{T} \frac{f(S, u)-f(S, T)}{(u-t)^{\alpha}} d u, & \\
& 0<\alpha<1 .
\end{aligned}
$$

For convenience, use the coordinate transform $\tau=T-t$ and denote

$$
V(S, \tau) \equiv f(S, T-\tau)=f(S, t) .
$$

Then we calculate

$$
\begin{aligned}
\frac{\partial^{\alpha} f}{\partial t^{\alpha}} & =\frac{1}{\Gamma(1-\alpha)} \frac{\partial}{\partial t} \int_{t}^{T} \frac{f(S, u)-f(S, T)}{(u-t)^{\alpha}} d u \\
& =\frac{1}{\Gamma(1-\alpha)} \frac{\partial}{\partial \tau} \int_{\tau}^{0} \frac{f(S, T-\theta)-f(S, T)}{(\tau-\theta)^{\alpha}} d \theta \\
& =\frac{-1}{\Gamma(1-\alpha)} \frac{\partial}{\partial \tau} \int_{0}^{\tau} \frac{V(S, \theta)-V(S, 0)}{(\tau-\theta)^{\alpha}} d \theta \\
& =-\frac{\partial}{\partial \tau} \int_{0}^{\tau}[V(S, \theta)-V(S, 0)] d \frac{(\tau-\theta)^{1-\alpha}}{1-\alpha} \\
& =-\frac{\partial}{\partial \tau} \int_{0}^{\tau} \frac{(\tau-\theta)^{1-\alpha}}{1-\alpha} \frac{\partial V(S, \theta)}{\partial \theta} d \theta \\
& =\frac{-1}{\Gamma(1-\alpha)} \int_{0}^{\tau} \frac{1}{(\tau-\theta)^{\alpha}} \frac{\partial V(S, \theta)}{\partial \theta} d \theta .
\end{aligned}
$$

Recall from [16] that the left-hand side Caputo fractional derivative is defined as

$$
{ }_{0}^{C} D_{\tau}^{\alpha} V(S, \tau) \equiv \frac{1}{\Gamma(1-\alpha)} \int_{0}^{\tau} \frac{1}{(\tau-\theta)^{\alpha}} \frac{\partial V(S, \theta)}{\partial \theta} d \theta .
$$


Then (6) gives that

$$
\frac{\partial^{\alpha} f}{\partial t^{\alpha}}=-{ }_{0}^{C} D_{\tau}^{\alpha} V(S, \tau)
$$

According to (3), (5), (8) and the facts

$$
\begin{gathered}
\frac{\partial f}{\partial S}=\frac{\partial V}{\partial S}, \\
\frac{\partial^{2} f}{\partial S^{2}}=\frac{\partial^{2} V}{\partial S^{2}},
\end{gathered}
$$

the valuation of American put option can be formulated as a time-fractional free-boundary problem:

$$
\begin{aligned}
{ }_{0}^{C} D_{\tau}^{\alpha} V(S, \tau) & =\frac{1}{2} \delta^{2} S^{2 \beta+2} \frac{\partial^{2} V}{\partial S^{2}}+(r-q) S \frac{\partial V}{\partial S}-r V \\
V(S, 0) & =\max (K-S, 0), \\
V\left(S_{f}(\tau), \tau\right) & =K-S_{f}(\tau) \\
\frac{\partial V\left(S_{f}(\tau), \tau\right)}{\partial S} & =-1 \\
\lim _{S \rightarrow \infty} V(S, \tau) & =0 .
\end{aligned}
$$

The main purpose of this paper is to solve problem (10)-(14).

\section{Laplace Transform Methods}

For $\lambda>0$, define the Laplace-Carson transform (LCT) as

$$
\widehat{V}(S, \lambda)=\int_{0}^{+\infty} V(S, \tau) \lambda e^{-\lambda \tau} d \tau:=\mathscr{L}_{\mathscr{C}}[V(S, \tau)](\lambda)
$$

The LCT is essentially the same as the Laplace transform (LT) and the relationship between LCT and LT is

$$
\mathscr{L}_{\mathscr{C}}[V(S, \tau)](\lambda)=\lambda \mathscr{L}[V(S, \tau)](\lambda)
$$

The reason of using the LCT is to simplify the notations in the later analysis. Using the Laplace transform formula for the Caputo fractional derivative (see (2.253) in [16]),

$$
\begin{aligned}
\mathscr{L}\left[{ }_{0}^{C} D_{\tau}^{\alpha} V(S, \tau)\right](\lambda)= & \lambda^{\alpha} \mathscr{L}[V(S, \tau)](\lambda) \\
& -\lambda^{\alpha-1} V(S, 0),
\end{aligned}
$$

and relationship (16), the LCT for ${ }_{0}^{C} D_{\tau}^{\alpha} V(S, \tau)$ is found as

$$
\begin{aligned}
\mathscr{L}_{\mathscr{C}} & {\left[{ }_{0}^{C} D_{\tau}^{\alpha} V(S, \tau)\right](\lambda) } \\
= & \lambda^{\alpha} \mathscr{L}_{\mathscr{C}}[V(S, \tau)](\lambda)-\lambda^{\alpha} V(S, 0) .
\end{aligned}
$$

Taking LCT to (10)-(14), we have

$$
\begin{aligned}
& \frac{1}{2} \delta^{2} S^{2 \beta+2} \frac{\partial^{2} \widehat{V}}{\partial S^{2}}+(r-q) S \frac{\partial \widehat{V}}{\partial S}-\left(\lambda^{\alpha}+r\right) \widehat{V} \\
& \quad+\lambda^{\alpha} \max (K-S, 0)=0, \\
& \widehat{V}\left(\widehat{S_{f}}(\lambda), \lambda\right)=K-\widehat{S_{f}}(\lambda), \\
& \frac{\widehat{V}\left(\widehat{S_{f}}(\lambda), \lambda\right)}{\partial S}=-1, \\
& \lim _{S \rightarrow \infty} \widehat{V}(S, \lambda)=0 .
\end{aligned}
$$

The solution of governing equation (19) is given by

$$
\widehat{V}(S, \lambda)
$$

$$
= \begin{cases}C_{11} \phi_{\lambda}(S)+C_{12} \psi_{\lambda}(S), & \text { when } S \in[K, \infty), \\ C_{21} \phi_{\lambda}(S)+C_{22} \psi_{\lambda}(S)+u_{\lambda}(S), & \text { when } S \in\left(\widehat{S_{f}}, K\right) .\end{cases}
$$

For the case $\beta=0$, the basis functions $\phi_{\lambda}(S)$ and $\psi_{\lambda}(S)$ have the following forms (the derivation is analogous to that in [17]):

$$
\begin{aligned}
& \phi_{\lambda}(S)=S^{\gamma_{-}}, \\
& \psi_{\lambda}(S)=S^{\gamma_{+}}, \\
& \quad \gamma_{ \pm}=-\gamma \pm \sqrt{\gamma^{2}+\frac{2\left(\lambda^{\alpha}+r\right)}{\delta^{2}}}, \gamma=\frac{r-q}{\delta^{2}}-\frac{1}{2} .
\end{aligned}
$$

By simple calculation, we have

$$
\begin{aligned}
\Lambda(S) & \equiv \xi(S) \omega_{\lambda}, \\
\xi(S) & =S^{-2 \gamma-1}, \\
\omega_{\lambda} & =2 \sqrt{\gamma^{2}+\frac{2\left(\lambda^{\alpha}+r\right)}{\delta^{2}} .}
\end{aligned}
$$

For the case $\beta \neq 0$, the functions $\phi_{\lambda}(S)$ and $\psi_{\lambda}(S)$ are given by (the derivation is similar to that in [18])

$\phi_{\lambda}(S)$

$$
= \begin{cases}S^{\beta+1 / 2} e^{\epsilon x(S) / 2} W_{k(\lambda), m}(x(S)), & \beta<0, \mu \neq 0, \\ S^{\beta+1 / 2} e^{\epsilon x(S) / 2} M_{k(\lambda), m}(x(S)), & \beta>0, \mu \neq 0, \\ S^{1 / 2} K_{\nu}\left(\sqrt{2\left(\lambda^{\alpha}+r\right)} z(S)\right), & \beta<0, \mu=0, \\ S^{1 / 2} I_{\nu}\left(\sqrt{2\left(\lambda^{\alpha}+r\right)} z(S)\right), & \beta>0, \mu=0,\end{cases}
$$

$\psi_{\lambda}(S)$

$$
= \begin{cases}S^{\beta+1 / 2} e^{\epsilon x(S) / 2} M_{k(\lambda), m}(x(S)), & \beta<0, \mu \neq 0, \\ S^{\beta+1 / 2} e^{\epsilon x(S) / 2} W_{k(\lambda), m}(x(S)), & \beta>0, \mu \neq 0, \\ S^{1 / 2} I_{\nu}\left(\sqrt{2\left(\lambda^{\alpha}+r\right)} z(S)\right), & \beta<0, \mu=0, \\ S^{1 / 2} K_{\nu}\left(\sqrt{2\left(\lambda^{\alpha}+r\right)} z(S)\right), & \beta>0, \mu=0,\end{cases}
$$


where $M_{k, m}(x)$ and $W_{k, m}(x)$ are the Whittaker functions, $I_{\nu}(x)$ and $K_{\nu}(x)$ are the modified Bessel functions (see, e.g., $[18])$, and

$$
\begin{aligned}
\mu & =r-q, \\
x(S) & =\frac{|\mu|}{\delta^{2}|\beta|} S^{-2 \beta}, \\
z(S) & =\frac{1}{\delta|\beta|} S^{-\beta}, \\
\epsilon & =\operatorname{sign}(\mu \beta), \\
m & =\frac{1}{4|\beta|}, \\
k(\lambda) & =\epsilon\left(\frac{1}{2}+\frac{1}{4 \beta}\right)-\frac{\lambda^{\alpha}+r}{2|\mu \beta|}, \\
\nu & =\frac{1}{2|\beta|} .
\end{aligned}
$$

Moreover, it can be calculated that

$$
\begin{aligned}
\Lambda(S) & =\xi(S) \omega_{\lambda}, \\
\xi(S) & =\exp \left(\frac{\mu}{\delta^{2} \beta} S^{-2 \beta}\right), \\
\omega_{\lambda} & = \begin{cases}\frac{2|r-q| \Gamma(2 m+1)}{\delta^{2} \Gamma(m-k+1 / 2)}, & r-q \neq 0, \\
|\beta|, & r-q=0 .\end{cases}
\end{aligned}
$$

Next, we give a particular solution $u_{\lambda}(S)$ and derive the nonlinear equation for $\widehat{S_{f}}(\lambda)$. By matching conditions (20)(22), we derive that

$$
\begin{aligned}
& u_{\lambda}(S)=-\frac{\lambda^{\alpha} S}{\lambda^{\alpha}+q}+\frac{\lambda^{\alpha} K}{\lambda^{\alpha}+r}, \\
& C_{12}=0, \\
& C_{11}=\frac{1}{a_{5} \Lambda(K)}\left[\left(a_{4} a_{5}-a_{3} a_{6}\right) b_{1}\right. \\
& \left.\quad+\left(a_{1} a_{6}-a_{2} a_{5}-\Lambda(K)\right) b_{2}-\Lambda(K)\right], \\
& C_{21}=\frac{1}{a_{5} \Lambda(K)}\left[-a_{3} a_{6} b_{1}+\left(a_{1} a_{6}-\Lambda(K)\right) b_{2}\right. \\
& -\Lambda(K)], \\
& C_{22}=\frac{1}{\Lambda(K)}\left[a_{3} b_{1}-a_{1} b_{2}\right],
\end{aligned}
$$

where

$$
\begin{aligned}
& a_{1}=\phi_{\lambda}(K), \\
& a_{2}=\psi_{\lambda}(K), \\
& a_{3}=\left.\frac{d \phi_{\lambda}(S)}{d S}\right|_{S=K}, \\
& a_{4}=\left.\frac{d \psi_{\lambda}(S)}{d S}\right|_{S=K}, \\
& a_{5}=\left.\frac{d \phi_{\lambda}(S)}{d S}\right|_{S=\widehat{S_{f}}(\lambda)}, \\
& a_{6}=\left.\frac{d \psi_{\lambda}(S)}{d S}\right|_{S=\widehat{S_{f}}(\lambda)}, \\
& b_{1}=u_{\lambda}(K), \\
& b_{2}=\frac{-\lambda^{\alpha}}{\lambda^{\alpha}+q} .
\end{aligned}
$$

Furthermore, letting $S=\widehat{S_{f}}(\lambda)$ in formula (23) and using conditions (20) and (21), we derive the nonlinear equation for $\widehat{S_{f}}(\lambda)$ :

$$
\begin{aligned}
A_{1} \Lambda\left(\widehat{S_{f}}(\lambda)\right)= & A_{2} \phi_{\lambda}\left(\widehat{S_{f}}(\lambda)\right) \\
& +\left.A_{3} \widehat{S_{f}}(\lambda) \frac{d \phi_{\lambda}(S)}{d S}\right|_{S=\widehat{S_{f}}(\lambda)} \\
& +\left.A_{4} \frac{d \psi_{\lambda}(S)}{d S}\right|_{S=\widehat{S_{f}}(\lambda)},
\end{aligned}
$$

where

$$
\begin{aligned}
A_{1}= & \left.\left(\frac{\lambda^{\alpha} K}{\lambda^{\alpha}+r}-\frac{\lambda^{\alpha} K}{\lambda^{\alpha}+q}\right) \frac{d \phi_{\lambda}(S)}{d S}\right|_{S=K} \\
& +\left.\frac{\lambda^{\alpha} \phi_{\lambda}(S)}{\lambda^{\alpha}+q}\right|_{S=K}, \\
A_{2}= & -\left.\frac{q \Lambda(S)}{\lambda^{\alpha}+q}\right|_{S=K}, \\
A_{3}= & \left.\frac{q \Lambda(S)}{\lambda^{\alpha}+q}\right|_{S=K}, \\
A_{4}= & -\left.\frac{r K \Lambda(S)}{\lambda^{\alpha}+r}\right|_{S=K} .
\end{aligned}
$$

Using the secant method to solve (31), we obtain $\widehat{S_{f}}(\lambda)$ for different values of $\lambda$. Finally, the optimal exercise boundary and put option price can be expressed in terms of the Laplace inversion:

$$
\begin{gathered}
S_{f}(\tau)=\mathscr{L}^{-1}\left[\frac{\widehat{S_{f}}(\lambda)}{\lambda}\right], \\
V(S, \tau)=\mathscr{L}^{-1}\left[\frac{\widehat{V}(S, \lambda)}{\lambda}\right] .
\end{gathered}
$$




\section{Finite Difference Methods}

Suppose $S_{\max }$ is a large enough positive number such that $V\left(S_{\max }, \tau\right) \approx 0$ for all $\tau \in[0, T]$. Define uniform time and space mesh

$$
\begin{aligned}
\Delta \tau & =\frac{T}{M}, \\
\tau_{k} & =k \Delta \tau, \quad k=0,1, \ldots, M, \\
\Delta S & =\frac{S_{\max }}{N}, \\
S_{j} & =j \Delta S, \quad j=0,1, \ldots, N .
\end{aligned}
$$

The time-fractional derivative ${ }_{0}^{C} D_{\tau}^{\alpha} V(S, \tau)$ at $S=S_{j}, \tau=\tau_{n}$ can be formulated as (see [19])

$$
\begin{aligned}
& \left.{ }_{0}^{C} D_{\tau}^{\alpha} V(S, \tau)\right|_{S=S_{j}, \tau=\tau_{n}} \\
& =\sum_{k=1}^{n} b_{k}^{(n)} \frac{V\left(S_{j}, \tau_{k}\right)-V\left(S_{j}, \tau_{k-1}\right)}{\Delta \tau}+O\left((\Delta \tau)^{2-\alpha}\right)
\end{aligned}
$$

with

$$
\begin{aligned}
b_{k}^{(n)} & =\frac{1}{\Gamma(1-\alpha)} \int_{\tau_{k-1}}^{\tau_{k}} \frac{d \omega}{\left(\tau_{n}-\omega\right)^{\alpha}} \\
& =\frac{1}{\Gamma(2-\alpha)}\left[\left(\tau_{n}-\tau_{k-1}\right)^{1-\alpha}-\left(\tau_{n}-\tau_{k}\right)^{1-\alpha}\right], \\
& k=1,2, \ldots, n-1, \\
b_{n}^{(n)} & =\frac{(\Delta \tau)^{1-\alpha}}{\Gamma(2-\alpha)} .
\end{aligned}
$$

We approximate the space derivatives by central difference:

$$
\begin{aligned}
\left.\frac{\partial^{2} V}{\partial S^{2}}\right|_{S=S_{j}, \tau=\tau_{n}} & \frac{V\left(S_{j-1}, \tau_{n}\right)-2 V\left(S_{j}, \tau_{n}\right)+V\left(S_{j+1}, \tau_{n}\right)}{(\Delta S)^{2}} \\
= & +O\left((\Delta S)^{2}\right), \\
\left.\frac{\partial V}{\partial S}\right|_{S=S_{j}, \tau=\tau_{n}} & V\left(S_{j+1}, \tau_{n}\right)-V\left(S_{j-1}, \tau_{n}\right) \\
= & \frac{2 \Delta S}{2 \Delta S}+O\left((\Delta S)^{2}\right) .
\end{aligned}
$$

Inserting (35) and (37) into PDE (10) and omitting the higher term $O\left((\Delta \tau)^{2-\alpha}\right)$ and $O\left((\Delta S)^{2}\right)$, we obtain the FDM scheme (with notation $V_{j}^{n} \approx V\left(S_{j}, \tau_{n}\right)$ ) as follows:

$$
c_{j-1} V_{j-1}^{n}+c_{j} V_{j}^{n}+c_{j+1} V_{j+1}^{n}=d_{j}^{n},
$$

where

$$
\begin{aligned}
c_{j-1} & =-\frac{\Delta \tau}{2(\Delta S)^{2}} \delta^{2} S_{j}^{2 \beta+2}+\frac{\Delta \tau}{2 \Delta S}(r-q) S_{j}, \\
c_{j} & =b_{n}^{(n)}+\frac{\Delta \tau}{(\Delta S)^{2}} \delta^{2} S_{j}^{2 \beta+2}, \\
c_{j+1} & =-\frac{\Delta \tau}{2(\Delta S)^{2}} \delta^{2} S_{j}^{2 \beta+2}-\frac{\Delta \tau}{2 \Delta S}(r-q) S_{j}, \\
d_{j}^{n} & =b_{n}^{(n)} V_{j}^{n-1}-\sum_{k=1}^{n-1} b_{k}^{(n)}\left(V_{j}^{k}-V_{j}^{k-1}\right) .
\end{aligned}
$$

The initial condition is $V_{j}^{0}=\max \left(K-S_{j}, 0\right)$, and the left-hand side boundary and the boundary conditions are specified by the following algorithm.

Differently from fixed boundary problems, how to determine the moving boundaries $S_{f}(\tau)$ is the key for pricing American option. We design a simple algorithm, namely, boundary-searching method, for solving time-fractional free-boundary problem (10)-(14).

\section{Boundary-Searching Algorithm}

Step 1. Let $S_{f}(0)=K$. Since $V_{j}^{0}=\max \left(K-S_{j}, 0\right)$, we determine $J\left(\tau_{0}\right)$ by

$$
J\left(\tau_{0}\right)=\min _{j}\left\{j \mid V_{j}^{0}=0\right\} .
$$

So we know $S_{J\left(\tau_{0}\right)-1} \leq S_{f}(0) \leq S_{J\left(\tau_{0}\right)}$.

Step 2. For $n=1, \ldots, M$

Step 2.1. Search backward for $J\left(\tau_{n}\right)$.

For $i=J\left(\tau_{n-1}\right)-1, J\left(\tau_{n-1}\right)-2, \ldots, 1,0$

Solve (38) with boundary conditions $V_{i}^{n}=K-S_{i}$ and $V_{N}^{n}=0$.

If the solutions $V_{i+1}^{n}$ and $V_{i}^{n}$ satisfy $\left(V_{i+1}^{n}-\right.$ $\left.V_{i}^{n}\right) / \Delta S \leq-1$, then

$$
J\left(\tau_{n}\right)=i+1 ; \text { Break; }
$$

End If

\section{End For}

Then we have that $S_{f}\left(\tau_{n}\right) \in\left[S_{J\left(\tau_{n}\right)-1}, S_{J\left(\tau_{n}\right)}\right]$. More accurately searching of $S_{f}\left(\tau_{n}\right)$ can be carried out in the next substep.

Step 2.2. Let $S_{f}^{\theta}\left(\tau_{n}\right) \equiv \theta S_{J\left(\tau_{n}\right)}+(1-\theta) S_{J\left(\tau_{n}\right)-1}, 0 \leq \theta \leq 1$. Then redefine the boundary conditions for (38) as

$$
\begin{gathered}
\frac{L_{-1}\left(S_{f}^{\theta}\left(\tau_{n}\right)\right)}{2(\Delta S)^{2}} V_{J\left(\tau_{n}\right)-1}^{n}-\frac{L_{0}\left(S_{f}^{\theta}\left(\tau_{n}\right)\right)}{(\Delta S)^{2}} V_{J\left(\tau_{n}\right)}^{n} \\
+\frac{L_{1}\left(S_{f}^{\theta}\left(\tau_{n}\right)\right)}{2(\Delta S)^{2}} V_{J\left(\tau_{n}\right)+1}^{n}=K-S_{f}^{\theta}\left(\tau_{n}\right)
\end{gathered}
$$


with Lagrange basis functions

$$
L_{\ell}(S)=\prod_{\substack{j=-1,0,1 \\ j \neq \ell}}\left[S-\left(J\left(\tau_{n}\right)+j\right) \Delta S\right], \quad \ell=-1,0,1
$$

Then using the solutions of (38) with the new boundary condition (41), we calculate the approximation

$$
\begin{aligned}
\frac{\partial}{\partial S} V\left(S_{f}^{\theta}\left(\tau_{n}\right), \tau_{n}\right) \approx & \frac{L_{-1}^{\prime}\left(S_{f}^{\theta}\left(\tau_{n}\right)\right)}{2(\Delta S)^{2}} V_{J\left(\tau_{n}\right)-1}^{n} \\
& -\frac{L_{0}^{\prime}\left(S_{f}^{\theta}\left(\tau_{n}\right)\right)}{(\Delta S)^{2}} V_{J\left(\tau_{n}\right)}^{n} \\
& +\frac{L_{1}^{\prime}\left(S_{f}^{\theta}\left(\tau_{n}\right)\right)}{2(\Delta S)^{2}} V_{J\left(\tau_{n}\right)+1}^{n} .
\end{aligned}
$$

We find the appropriate value of $0 \leq \theta \leq 1$ such that

$$
\frac{\partial}{\partial S} V\left(S_{f}^{\theta}\left(\tau_{n}\right), \tau_{n}\right) \approx-1
$$

Step 3. Output early exercise boundaries $S_{f}^{\theta}\left(\tau_{n}\right)$ and American put option prices $V_{j}^{n}$ (for $j=0,1, \ldots, J\left(\tau_{n}\right)-1, V_{j}^{n}=K-S_{j}$ and for $j=J\left(\tau_{n}\right), \ldots, N, V_{j}^{n}$ are obtained from FDM (38) with the new boundary condition (41)).

Remark 1. (a) The boundary-searching method is based on the fact that the early exercise boundary $S_{f}(\tau)$ is monotonically decreasing in $\tau$ and the derivative $\partial V / \partial S$ is monotonically increasing with respect to $S$. (b) Note that the left-hand side of (41) is just the quadratic Lagrange approximation of $V\left(S_{f}^{\theta}\left(\tau_{n}\right), \tau_{n}\right)$. The role of Step 2.2 is to numerically search a value $S_{f}\left(\tau_{n}\right) \in\left[S_{J\left(\tau_{n}\right)-1}, S_{J}\left(\tau_{n}\right)\right]$ such that

$$
\begin{aligned}
\frac{\partial}{\partial S} V\left(S_{f}\left(\tau_{n}\right), \tau_{n}\right) & =-1, \\
V\left(S_{f}\left(\tau_{n}\right), \tau_{n}\right) & =K-S_{f}\left(\tau_{n}\right) .
\end{aligned}
$$

In fact, the boundary-searching method still works without Step 2.2; however, Step 2.2 can lead to more accurate results.

\section{Numerical Examples}

In this section, we implement and compare the Laplace transform method and the boundary-searching finite difference method (FDM). The Laplace inversion is calculated by GaverWynn-Rho (GWR) algorithm in [20]. The mesh parameters are taken as $M=200, N=800$, and $S_{\max }=200$ for FDM. The volatility at time $t=0$ is defined by $\sigma_{0} \equiv \delta S_{0}^{\beta}$.

Table 1 lists the computational values of American put

\begin{tabular}{|c|c|c|c|c|}
\hline \multicolumn{5}{|c|}{$r=0.05, q=0, T=3, S_{0}=40, K=40, \beta=0$} \\
\hline \multirow{2}{*}{$\alpha$} & \multicolumn{2}{|c|}{$\sigma_{0}=0.1$} & \multicolumn{2}{|c|}{$\sigma_{0}=0.2$} \\
\hline & LTM & FDM & LTM & FDM \\
\hline 1.0 & 1.2189 & 1.2362 & 3.4116 & 3.4792 \\
\hline 0.9 & 1.1771 & 1.1912 & 3.2651 & 3.3157 \\
\hline 0.7 & 1.0959 & 1.1028 & 2.9817 & 3.0071 \\
\hline 0.4 & 0.9778 & 0.9793 & 2.5770 & 2.5829 \\
\hline 0.2 & 0.9005 & 0.9002 & 2.3184 & 2.3191 \\
\hline CPU time $(\mathrm{s})$ & 1.65 & 234.68 & 1.73 & 241.34 \\
\hline \multicolumn{5}{|c|}{$r=0.05, q=0, T=3, S_{0}=40, K=40, \beta=-1$} \\
\hline \multirow{2}{*}{$\alpha$} & \multicolumn{2}{|c|}{$\sigma_{0}=0.1$} & \multicolumn{2}{|c|}{$\sigma_{0}=0.2$} \\
\hline & LTM & FDM & LTM & FDM \\
\hline 1.0 & 1.1877 & 1.2020 & 3.3325 & 3.3834 \\
\hline 0.9 & 1.1485 & 1.1604 & 3.1918 & 3.2297 \\
\hline 0.7 & 1.0722 & 1.0802 & 2.9208 & 2.9400 \\
\hline 0.4 & 0.9609 & 0.9657 & 2.5347 & 2.5397 \\
\hline 0.2 & 0.8877 & 0.8922 & 2.2876 & 2.2898 \\
\hline CPU time (s) & 1.53 & 239.21 & 1.98 & 247.22 \\
\hline
\end{tabular}
options. Columns entitled "LTM" and "FDM" represent the Laplace transform method and the boundary-searching FDM, respectively. For $\alpha=1$, the time-fractional derivative reduces to the first-order derivative in time and the problem becomes the classical American option pricing problem.
TABle 1: Prices of American put option at $\tau=3$.

From Table 1, we can see that when $\alpha=1$ the results are consistent with the prices listed in [12]. At time to maturity $\tau=3$, one could observe that the option prices are decreasing as $\alpha$ is decreasing. Moreover, the LTM takes much less CPU time than the FDM as observed from Table 1.

In Figure 1, we plot the early exercise boundaries computed by LTM and FDM for the cases of $\alpha=0.7$ and $\alpha=0.4$. The numbers of mesh nodes are taken as $M=50, N=200$ for FDM. It can be seen that all the boundaries at different obtained by LTM are very close time $\tau$ obtained by LTM are very close to those by FDM. Figure 2 illustrates the effect of different time-fractional derivative order $\alpha$ on the early exercise boundaries and option prices. It can be seen from Figure 2(a) that when the value of $\alpha$ is decreasing, the exercise region will be shrunk in the region of time to maturity $\tau$ close to 0 and will be enlarged in the region of $\tau$ close to $T$. From Figure 2(b) we observe that the time-fractional derivatives have little effect on the option price for the cases of deep-in-the-money $\left(S_{0} \ll K\right)$ and deep-out-the-money $\left(S_{0} \gg K\right)$ and have significant effect near to on-the-money $\left(S_{0} \approx K\right)$.

\section{Conclusions}

There have been increasing applications of fractional PDEs in the field of option pricing. In the history most focuses have been on the valuation of options without early exercise features. In current work we study the American option pricing with time-fractional PDEs. The option pricing problem is formulated into a time-fractional PDE with free boundary. Two methods, namely, Laplace transform method (LTM) and finite difference method (FDM), are proposed to solve the time-fractional free-boundary problem. Numerical 

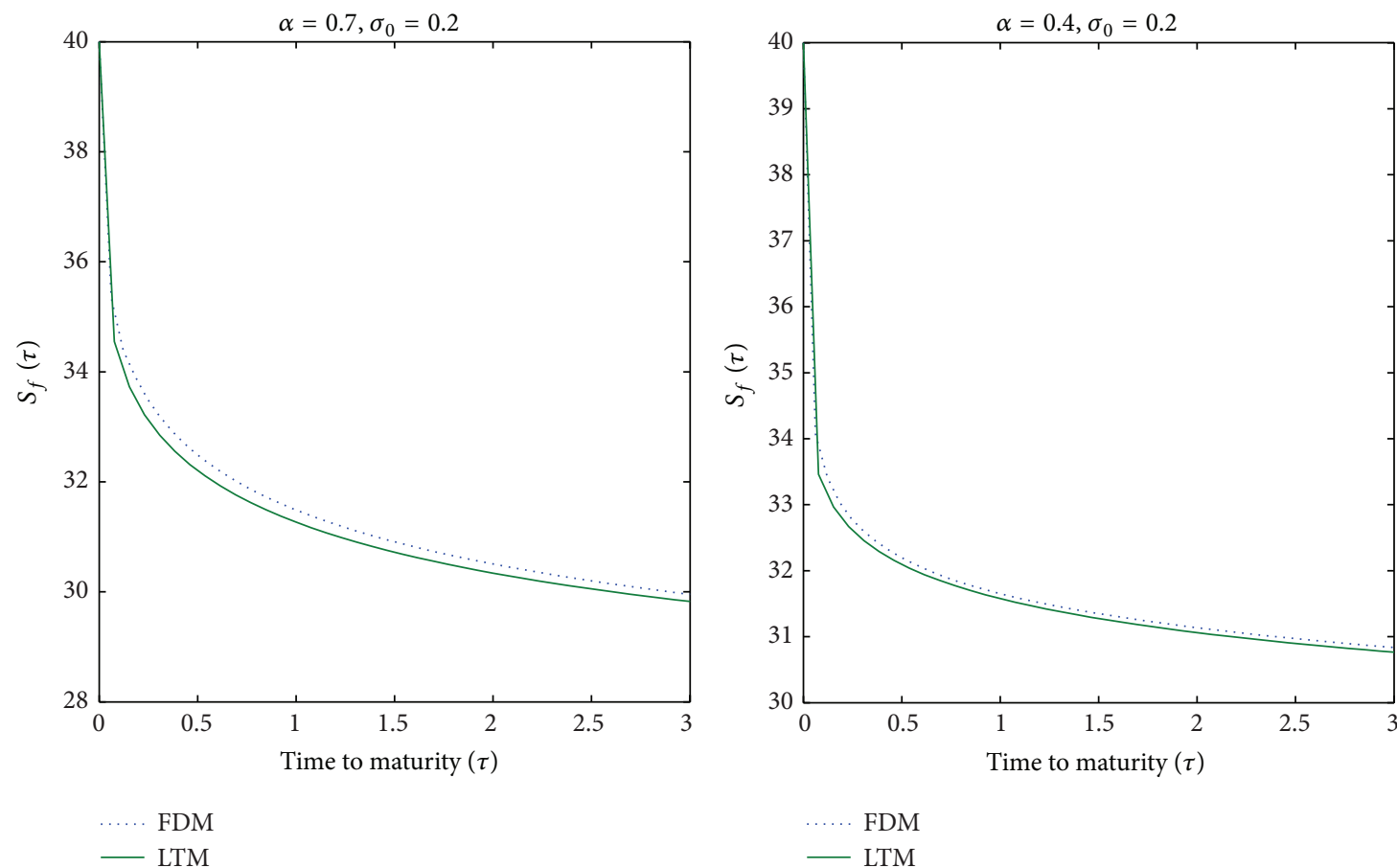

FIGURE 1: Early exercise boundaries computed by LTM and FDM.

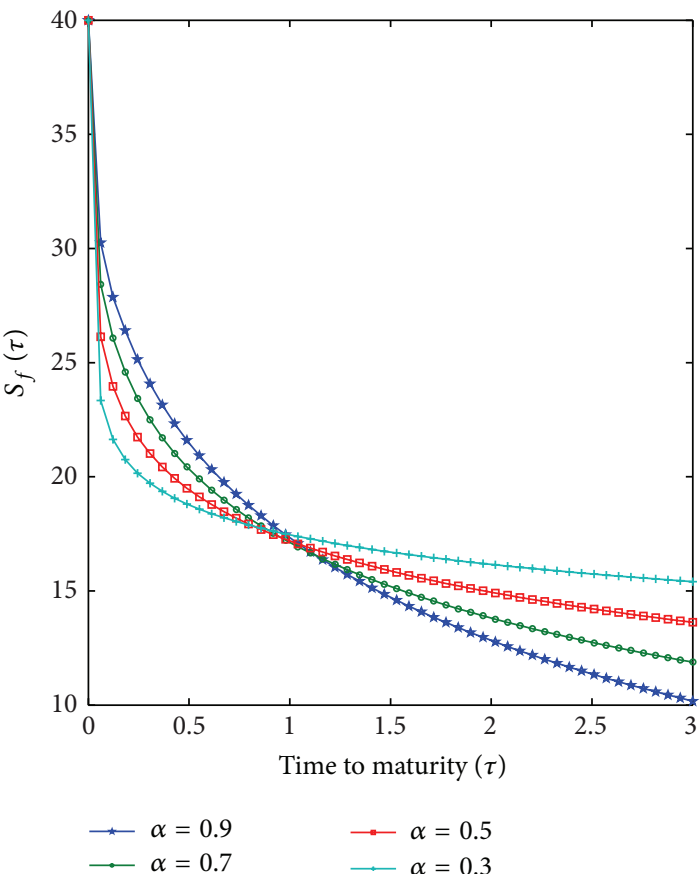

(a)

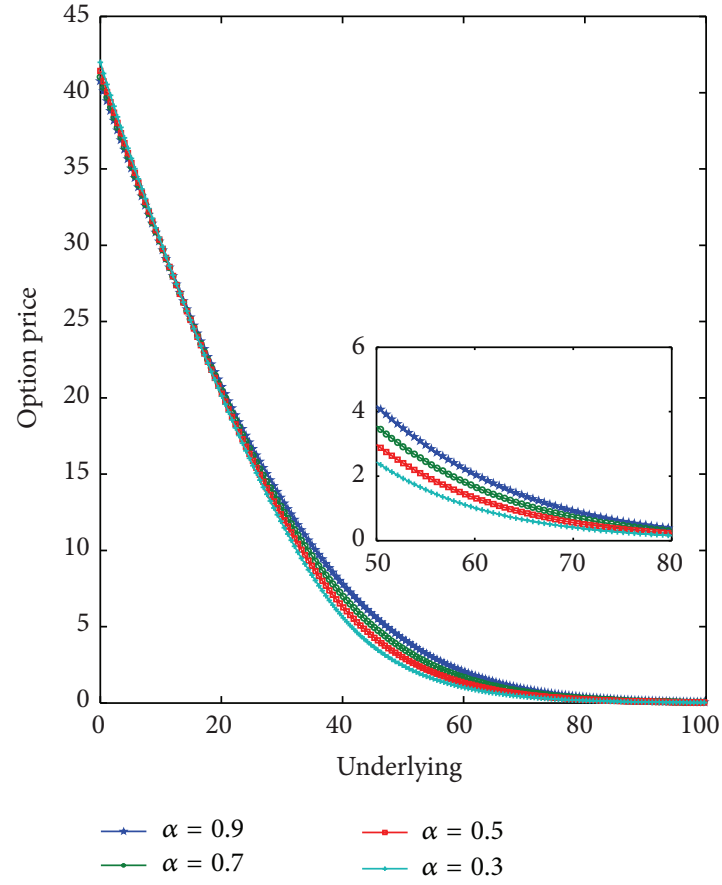

(b)

FIGURE 2: Early exercise boundaries (a) and option prices at $\tau=T=3$ (b) with different $\alpha$ and fixed $\sigma_{0}=0.4, \beta=-1, r=0.05, q=0$, and $K=40$.

examples show that the LTM is more efficient than the FDM. The methods in this paper are quite different to the predictor-corrector approach for pricing American options under space-fractional derivatives in [11]. The extension of the methods in this paper to the American option pricing under space-fractional models will be left for the future work. In addition it will be also interesting to extend the methods to the newly established models [21,22]. 


\section{Conflict of Interests}

The authors declare that there is no conflict of interests regarding the publication of this paper.

\section{Acknowledgments}

The work was supported by the Fundamental Research Funds for the Central Universities (Grant nos. 15CX141110 and JBK1307012) and National Natural Science Foundation of China (Grant no. 11471137).

\section{References}

[1] D. Baleanu, K. Diethelm, E. Scalas, and J. J. Trujillo, Fractional Calculus: Models and Numerical Methods, World Scientific Publishers, London, UK, 2012.

[2] P. Carr and L. Wu, "The finite moment log stable process and option pricing," The Journal of Finance, vol. 58, no. 2, pp. 597626, 2003.

[3] Á. Cartea and D. del-Castillo-Negrete, "Fractional diffusion models of option prices in markets with jumps," Physica A: Statistical Mechanics and Its Applications, vol. 374, no. 2, pp. 749-763, 2007.

[4] W. Chen, X. Xu, and S.-P. Zhu, "Analytically pricing Europeanstyle options under the modified Black-Scholes equation with a spatial-fractional derivative," Quarterly of Applied Mathematics, vol. 72, no. 3, pp. 597-611, 2014.

[5] W. Wyss, “The fractional Black-Scholes equation," Fractional Calculus \& Applied Analysis, vol. 3, no. 1, pp. 51-61, 2000.

[6] S. Kumar, A. Yildirim, Y. Khan, H. Jafari, K. Sayevand, and L. Wei, "Analytical solution of fractional Black-Scholes European option pricing equation by using Laplace transform," Fractional Calculus and Applied Analysis, vol. 2, pp. 1-9, 2012.

[7] Á. Cartea, "Derivatives pricing with marked point processes using tick-by-tick data," Quantitative Finance, vol. 13, no. 1, pp. 111-123, 2013.

[8] G. Jumarie, "Stock exchange fractional dynamics defined as fractional exponential growth driven by (usual) Gaussian white noise. Application to fractional Black-Scholes equations," Insurance: Mathematics and Economics, vol. 42, no. 1, pp. 271-287, 2008.

[9] J.-R. Liang, J. Wang, W.-J. Zhang, W.-Y. Qiu, and F.-Y. Ren, “The solution to a bifractional Black-Scholes-Merton differential equation," International Journal of Pure and Applied Mathematics, vol. 58, no. 1, pp. 99-112, 2010.

[10] W. Chen, X. Xu, and S.-P. Zhu, "Analytically pricing double barrier options based on a time-fractional Black-Scholes equation," Computers \& Mathematics with Applications, vol. 69, no. 12, pp. 1407-1419, 2015.

[11] W. Chen, X. Xu, and S.-P. Zhu, "A predictor-corrector approach for pricing American options under the finite moment logstable model," Applied Numerical Mathematics, vol. 97, pp. 1529, 2015.

[12] H. Y. Wong and J. Zhao, "Valuing American options under the CEV model by Laplace-Carson transforms," Operations Research Letters, vol. 38, no. 5, pp. 474-481, 2010.

[13] K. Muthuraman, "A moving boundary approach to American option pricing," Journal of Economic Dynamics \& Control, vol. 32, no. 11, pp. 3520-3537, 2008.
[14] A. Chockalingam and K. Muthuraman, "An approximate moving boundary method for American option pricing," European Journal of Operational Research, vol. 240, no. 2, pp. 431-438, 2015.

[15] J. Cox, "Notes on option pricing I: constant elasticity of variance diffusions," The Journal of Portfolio Management, vol. 22, pp. 1517, 1996.

[16] I. Podlubny, Fractional Differential Equations, Academic Press, 1999.

[17] P. Carr, "Randomization and the American put," Review of Financial Studies, vol. 11, no. 3, pp. 597-626, 1998.

[18] D. Davydov and V. Linetsky, "Pricing and hedging pathdependent options under the CEV process," Management Science, vol. 47, no. 7, pp. 949-965, 2001.

[19] Y. Jiang and J. Ma, "Moving finite element methods for time fractional partial differential equations," Science China Mathematics, vol. 56, no. 6, pp. 1287-1300, 2013.

[20] P. P. Valkó and S. Vajda, "Inversion of noise-free Laplace transforms: towards a standardized set of test problems," Inverse Problems in Engineering, vol. 10, no. 5, pp. 467-483, 2002.

[21] C. Huang, X. Ma, and Q. Lan, "An empirical study on listed company's value of cash holdings: an information asymmetry perspective," Discrete Dynamics in Nature and Society, vol. 2014, Article ID 897278, 12 pages, 2014.

[22] C. Huang, X. Yang, X. G. Yang, and H. Sheng, "An empirical study of the effect of investor sentiment on returns of different industries," Mathematical Problems in Engineering, vol. 2014, Article ID 545723, 11 pages, 2014. 


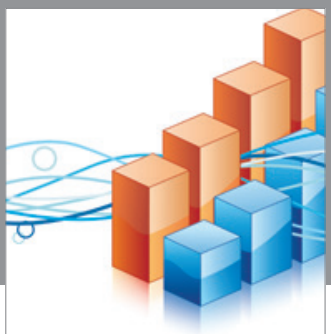

Advances in

Operations Research

vatem alat4

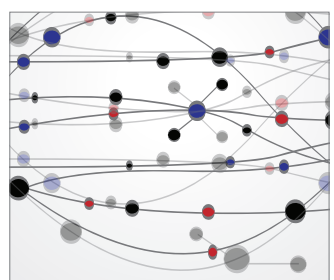

\section{The Scientific} World Journal
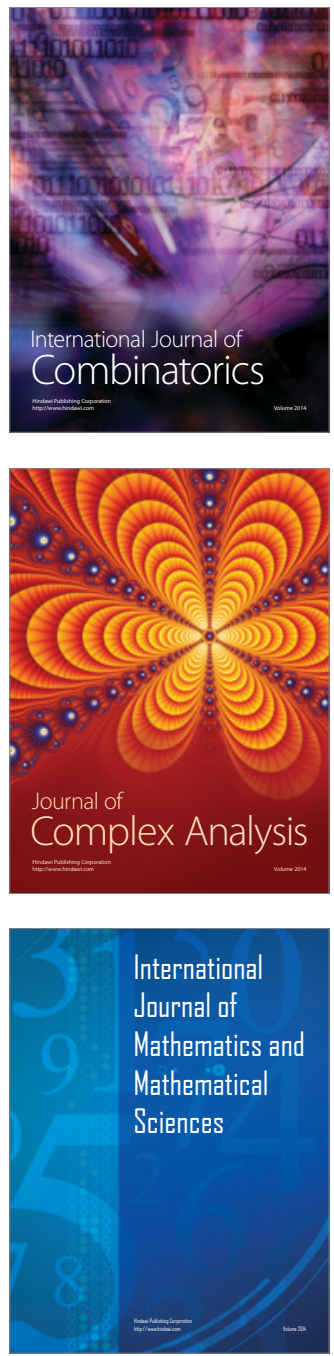
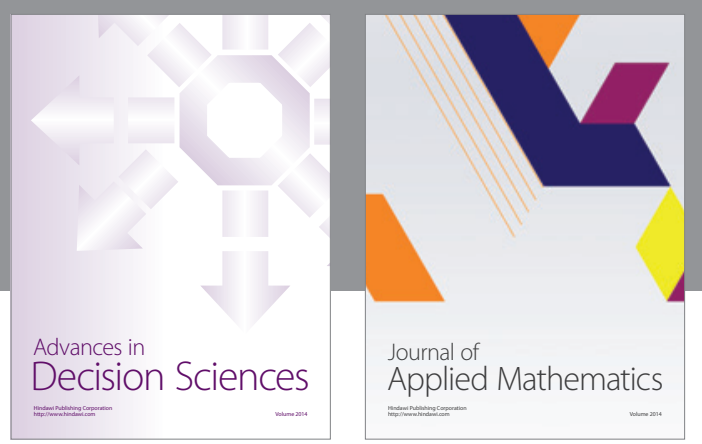

Algebra

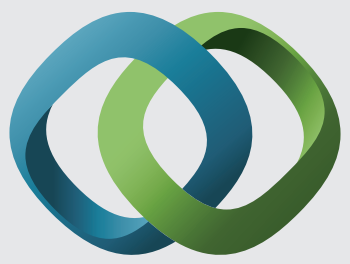

\section{Hindawi}

Submit your manuscripts at

http://www.hindawi.com
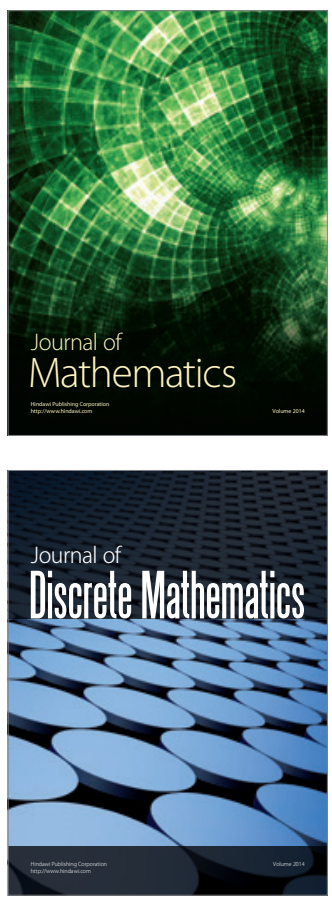

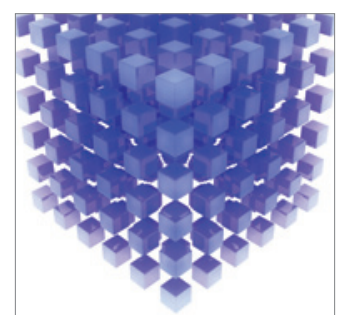

Mathematical Problems in Engineering
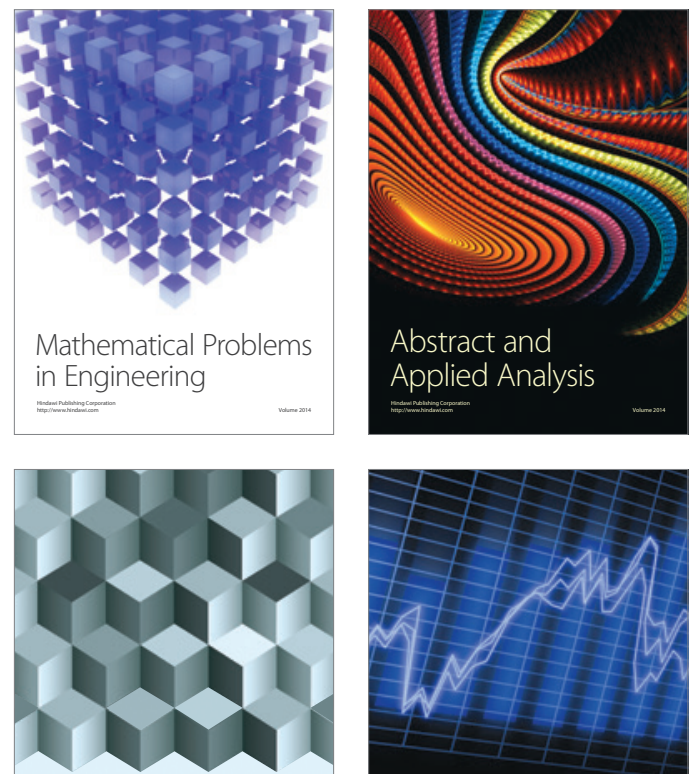

Journal of

Function Spaces

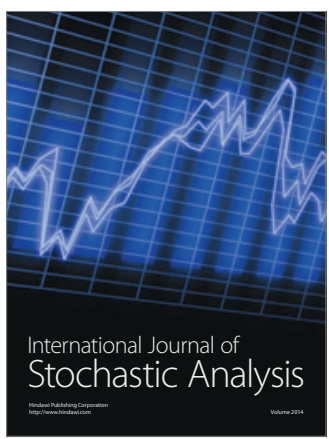

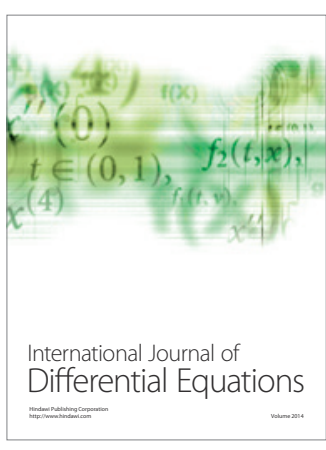
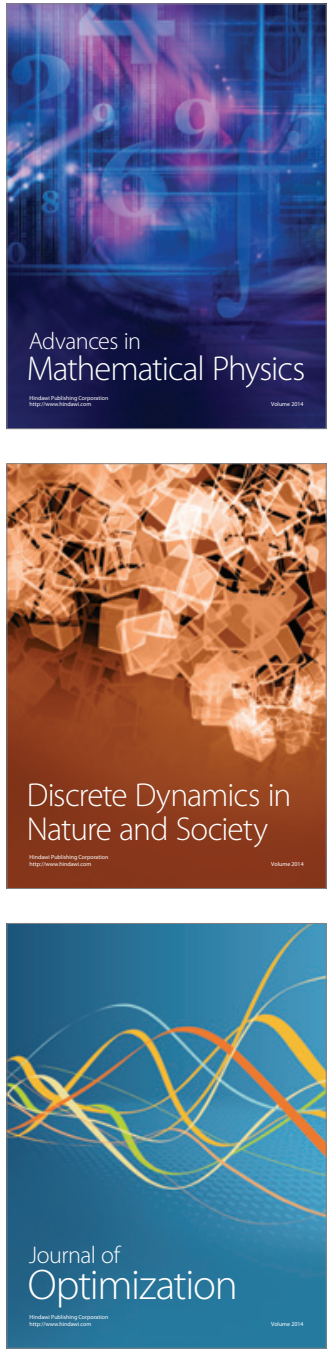